TRANSACTIONS OF THE

AMERICAN MATHEMATICAL SOCIETY

Volume 354, Number 6, Pages 2461-2474

S 0002-9947(02)02970-7

Article electronically published on February 14, 2002

\title{
HYPONORMALITY OF TRIGONOMETRIC TOEPLITZ OPERATORS
}

\author{
IN SUNG HWANG AND WOO YOUNG LEE
}

Dedicated to Professor Yong Tae Kim on his 65th birthday

\begin{abstract}
In this paper we establish a tractable and explicit criterion for the hyponormality of arbitrary trigonometric Toeplitz operators, i.e., Toeplitz operators $T_{\varphi}$ with trigonometric polynomial symbols $\varphi$. Our criterion involves the zeros of an analytic polynomial $f$ induced by the Fourier coefficients of $\varphi$. Moreover the rank of the selfcommutator of $T_{\varphi}$ is computed from the number of zeros of $f$ in the open unit disk $\mathbb{D}$ and in $\mathbb{C} \backslash \overline{\mathbb{D}}$ counting multiplicity.
\end{abstract}

\section{INTRODUCTION}

A bounded linear operator $A$ on a Hilbert space $\mathfrak{H}$ with inner product $(\cdot, \cdot)$ is said to be hyponormal if its selfcommutator $\left[A^{*}, A\right]=A^{*} A-A A^{*}$ induces a positive semidefinite quadratic form on $\mathfrak{H}$ via $\xi \mapsto\left(\left[A^{*}, A\right] \xi, \xi\right)$, for $\xi \in \mathfrak{H}$. Recall that given $\varphi \in L^{\infty}(\mathbb{T})$, the Toeplitz operator with symbol $\varphi$ is the operator $T_{\varphi}$ on the Hardy space $H^{2}(\mathbb{T})$ of the unit circle $\mathbb{T}=\partial \mathbb{D}$ in the complex plane $\mathbb{C}$ defined by $T_{\varphi} f=P(\varphi \cdot f)$, where $f \in H^{2}(\mathbb{T})$ and $P$ denotes the orthogonal projection that maps $L^{2}(\mathbb{T})$ onto $H^{2}(\mathbb{T})$.

An elegant theorem of C. Cowen [Co2] characterizes the hyponormality of Toeplitz operators $T_{\varphi}$ on $H^{2}(\mathbb{T})$ by properties of the symbol $\varphi \in L^{\infty}(\mathbb{T})$. Normal Toeplitz operators were characterized by a property of their symbols in the early 1960 's by A. Brown and P. Halmos $[\mathrm{BH}]$, and 25 years passed before the exact nature of the relationship between the symbol $\varphi \in L^{\infty}(\mathbb{T})$ and the positivity of the selfcommutator $\left[T_{\varphi}^{*}, T_{\varphi}\right]$ was understood via Cowen's theorem [Co2]. As Cowen notes in his survey paper [Co1], the intensive study of subnormal Toeplitz operators in the 1970's and early 80 's is one explanation for the relatively late appearance of the sequel to the Brown-Halmos work. In fact, it remains still open to characterize subnormality of Toeplitz operators in terms of their symbols though C. Cowen and J. Long [CoL] answered in the negative to Problem 5 of Halmos's 1970 lectures "Ten problems in Hilbert space" (cf. [Ha1, Ha2]): Is every subnormal Toeplitz operator either normal or analytic? The characterization of hyponormality in Co2] requires one to solve a certain functional equation in the unit ball of $H^{\infty}(\mathbb{T})$. Here we shall employ an equivalent variant of Cowen's theorem that was first proposed by T. Nakazi and K. Takahashi $\left[\mathrm{NT}\right.$. Suppose that $\varphi \in L^{\infty}(\mathbb{T})$ is arbitrary and

Received by the editors October 19, 2000.

2000 Mathematics Subject Classification. Primary 47B20, 47B35.

Key words and phrases. Trigonometric Toeplitz operators, hyponormal operators.

This work was partially supported by KOSEF research project No. R01-2000-00003. 
consider the following subset of the closed unit ball of $H^{\infty}(\mathbb{T})$ :

$$
\mathcal{E}(\varphi)=\left\{k \in H^{\infty}(\mathbb{T}):\|k\|_{\infty} \leq 1 \text { and } \varphi-k \bar{\varphi} \in H^{\infty}(\mathbb{T})\right\} .
$$

The criterion is that $T_{\varphi}$ is hyponormal if and only if the set $\mathcal{E}(\varphi)$ is nonempty [Co2], [NT]. This theorem is referred to as the Cowen's theorem. Cowen's method is to recast the operator-theoretic problem of hyponormality for Toeplitz operators into the problem of finding a solution of a certain functional equation involving its symbol. This approach has been put to use in the works [CuL], [FL1], FL2], HKL, $, \mathrm{NT}, \mathrm{Zhu}$ to study hyponormal Toeplitz operators on $H^{2}(\mathbb{T})$. An abstract version of Cowen's method has been developed in [Gu].

If $\varphi$ is a trigonometric polynomial of the form $\varphi(z)=\sum_{n=-m}^{N} a_{n} z^{n}$, where $a_{-m}$ and $a_{N}$ are nonzero, then the nonnegative integers $N$ and $m$ denote the analytic and co-analytic degrees of $\varphi$. If a function $k \in H^{\infty}(\mathbb{T})$ satisfies $\varphi-k \bar{\varphi} \in H^{\infty}(\mathbb{T})$, then $k$ necessarily satisfies

$$
k \sum_{n=1}^{N} \overline{a_{n}} z^{-n}-\sum_{n=1}^{m} a_{-n} z^{-n} \in H^{\infty}(\mathbb{T}) .
$$

From (1) one computes the Fourier coefficients $\hat{k}(0), \ldots, \hat{k}(N-1)$ of $k$ to be $\hat{k}(n)=$ $c_{n}$, for $n=0,1, \ldots, N-1$, where $c_{0}, c_{1}, \ldots, c_{N-1}$ are determined uniquely from the coefficients of $\varphi$ by the recurrence relation

$$
\begin{aligned}
& c_{0}=c_{1}=\cdots=c_{N-m-1}=0, \\
& \text { (2) } c_{N-m}=\frac{a_{-m}}{\overline{a_{N}}}, \\
& c_{n}=\left(\overline{a_{N}}\right)^{-1}\left(a_{-N+n}-\sum_{j=N-m}^{n-1} c_{j} \overline{a_{N-n+j}}\right), \text { for } n=N-m+1, \cdots, N-1,
\end{aligned}
$$

or in matrix form,

$$
\begin{aligned}
& c_{0}=c_{1}=\cdots=c_{N-m-1}=0, \\
& \left(\begin{array}{c}
\overline{c_{N-m}} \\
\overline{c_{N-m+1}} \\
\vdots \\
\overline{c_{N-1}}
\end{array}\right)=\left(\begin{array}{ccccc}
a_{N-m+1} & a_{N-m+2} & \ldots & a_{N-1} & a_{N} \\
a_{N-m+2} & a_{N-m+3} & \ldots & a_{N} & 0 \\
\vdots & \vdots & \ddots & \vdots & \vdots \\
a_{N} & 0 & \ldots & 0 & 0
\end{array}\right)^{-1}\left(\begin{array}{c}
\overline{a_{-1}} \\
\overline{a_{-2}} \\
\vdots \\
\overline{a_{-m}}
\end{array}\right) .
\end{aligned}
$$

Thus $k_{p}(z):=\sum_{j=N-m}^{N-1} c_{j} z^{j}$ is the unique analytic polynomial of degree less than $N$ satisfying $\varphi-k \bar{\varphi} \in H^{\infty}$. However despite the fact that the recurrence relation (3) can always be solved uniquely to produce an analytic polynomial $k_{p}$ satisfying $\varphi-k_{p} \bar{\varphi} \in H^{\infty}$, the polynomial $k_{p}$ need not be contained in the set $\mathcal{E}(\varphi)$, even if $\mathcal{E}(\varphi)$ is known to be nonempty. Thus the problem of finding a solution in $\mathcal{E}(\varphi)$ is to find a function $k$ in the closed unit ball of $H^{\infty}(\mathbb{T})$ interpolating $k_{p}$. Recently K. Zhu [Zhu] has adopted a method based on the classical interpolation theorems of I. Schur Sch to obtain an abstract characterization of those trigonometric polynomial symbols that correspond to hyponormal Toeplitz operators. Furthermore, he was able to use this characterization to give explicit necessary and sufficient conditions for hyponormality in terms of the coefficients of the polynomial $\varphi$ whenever $m \leq 3$. Also in [FL1], using the preceding consideration, the hyponormality of $T_{\varphi}$ was completely characterized for the cases where $\varphi$ has outer coefficients of the same modulus, i.e., $\left|a_{-m}\right|=\left|a_{N}\right|$. However, with polynomials of higher degree 
with $\left|a_{-m}\right|<\left|a_{N}\right|$, the analogous explicit necessary and sufficient conditions (via properties of coefficients) are not known. Indeed the case of arbitrary trigonometric polynomial $\varphi$, though solved in principle by Cowen's theorem or Schur's algorithm, is in practice very complicated.

The goal of the present paper is to establish a tractable and explicit criterion for hyponormality of arbitrary trigonometric Toeplitz operators, i.e., Toeplitz operators with trigonometric polynomial symbols. In Section 2 we discuss preliminary results and present the main theorem - a criterion for the hyponormality of trigonometric Toeplitz operators. In Section 3 we provide auxiliary lemmas which are needed for proving the main theorem and Section 4 is devoted to the proof of the main theorem. In Section 5 we give remarks and examples which illustrate the main theorem.

\section{Preliminaries and the Criterion}

We first review Schur's algorithm determining hyponormality of trigonometric Toeplitz operators. Suppose that $k(z)=\sum_{j=0}^{\infty} c_{j} z^{j}$ is in the closed unit ball of $H^{\infty}(\mathbb{T})$. Let $k_{0}:=k$. Define by induction a sequence $\left\{k_{n}\right\}$ of functions in the closed unit ball of $H^{\infty}(\mathbb{T})$ as follows:

$$
k_{n+1}(z)=\frac{k_{n}(z)-k_{n}(0)}{z\left(1-\overline{k_{n}(0)} k_{n}(z)\right)}, \quad|z|<1, n=0,1,2, \cdots .
$$

Then $k_{n}(0)$ only depends on the coefficients $c_{0}, c_{1}, \cdots, c_{n}$. We write $k_{n}(0)=$ $\Phi_{n}\left(c_{0}, \cdots, c_{n}\right)$ for $n=0,1,2, \cdots$, where $\Phi_{n}$ is a function of $n+1$ complex variables. We call the $\Phi_{n}$ 's Schur's functions. Then Zhu's theorem is as follows:

Zhu's Theorem ([Zhu $]$ ). If $\varphi(z)=\sum_{n=-m}^{N} a_{n} z^{n}$, where $a_{N} \neq 0$ and if $c_{0}, \cdots$, $c_{N-1}$ are given by (3), then the following statements are equivalent.

1. $T_{\varphi}$ is a hyponormal operator.

2. $\left|\Phi_{n}\left(c_{0}, \cdots, c_{n}\right)\right| \leq 1$ for every $n=0,1, \cdots, N-1$.

As we noted in the introduction, if $k(z)=\sum_{j=0}^{\infty} c_{j} z^{j}$ is a function in $H^{\infty}(\mathbb{T})$, then $\varphi-k \bar{\varphi} \in H^{\infty}(\mathbb{T})$ if and only if $c_{0}, \cdots, c_{N-1}$ are given by (3). So by Cowen's theorem, if $c_{0}, \cdots, c_{N-1}$ are given by (3) then the hyponormality of $T_{\varphi}$ is equivalent to the existence of a function $k \in H^{\infty}(\mathbb{T})$ satisfying

(i) $\widehat{k}(j)=c_{j}, \quad j=0, \cdots, N-1$;

(ii) $\|k\|_{\infty} \leq 1$.

This is exactly the classical interpolation theorem solving the so-called CarathéodorySchur interpolation problem (CSIP). CSIP is analyzed by Schur's functions (cf. Sch] ): CSIP is solvable if and only if $\left|\Phi_{n}\left(c_{0}, \cdots, c_{n}\right)\right| \leq 1$ for every $n=0,1, \cdots, N-$ 1. Thus Zhu's theorem follows at once. By a straightforward calculation we can see that

$$
\Phi_{0}\left(c_{0}\right)=c_{0}, \quad \Phi_{1}\left(c_{0}, c_{1}\right)=\frac{c_{1}}{1-\left|c_{0}\right|^{2}}, \quad \Phi_{2}\left(c_{0}, c_{1}, c_{2}\right)=\frac{c_{2}\left(1-\left|c_{0}\right|^{2}\right)+\overline{c_{0}} c_{1}^{2}}{\left(1-\left|c_{0}\right|^{2}\right)^{2}-\left|c_{1}\right|^{2}}
$$

so that for example, if $\varphi(z)=\sum_{n=-2}^{2} a_{n} z^{n}$ then $T_{\varphi}$ is hyponormal if and only if

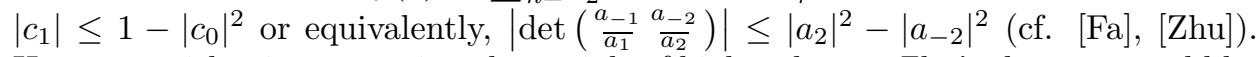
However, with trigonometric polynomials of higher degree, Zhu's theorem would be too complicated to be of much value because no closed-form for Schur's function $\Phi_{n}$ is known. 
The following theorem due to T. Nakazi and K. Takahashi [NT] provides useful information for $\mathcal{E}(\varphi)$ corresponding to hyponormal trigonometric Toeplitz operators $T_{\varphi}$

Nakazi-Takahashi Theorem ([NT]). A Toeplitz operator $T_{\varphi}$ is hyponormal and the rank of the selfcommutator $\left[T_{\varphi}^{*}, T_{\varphi}\right]$ is finite (e.g., $\varphi$ is a trigonometric polynomial) if and only if there exists a finite Blaschke product $k \in \mathcal{E}(\varphi)$ of the form

$$
k(z)=e^{i \theta} \prod_{j=1}^{n} \frac{z-\beta_{j}}{1-\overline{\beta_{j}} z} \quad\left(\left|\beta_{j}\right|<1 \text { for } j=1, \cdots, n\right) .
$$

such that $\operatorname{deg}(k)=\operatorname{rank}\left[T_{\varphi}^{*}, T_{\varphi}\right]$, where $\operatorname{deg}(k)$ denotes the degree of $k$ - meaning the number of zeros of $k$ in the open unit disk $\mathbb{D}$.

Before continuing further, we record here results on the hyponormality of trigonometric Toeplitz operators, which have been recently developed in the literature.

Proposition 1. Suppose that $\varphi(z)=\sum_{n=-m}^{N} a_{n} z^{n}$, where $a_{-m}$ and $a_{N}$ are nonzero.

(i) If $T_{\varphi}$ is hyponormal, then $m \leq N,\left|a_{-m}\right| \leq\left|a_{N}\right|$ and $N-m \leq \operatorname{rank}\left[T_{\varphi}^{*}, T_{\varphi}\right] \leq$ $N$. Furthermore, the hyponormality of $T_{\varphi}$ is independent of the particular values of the Fourier coefficients $a_{0}, a_{1}, \cdots, a_{N-m}$ of $\varphi$.

(ii) If $\varphi:=\bar{g}+f$, where $f$ and $g$ are in $H^{\infty}(\mathbb{T})$, and if $\widetilde{\varphi}:=\bar{g}+T_{\bar{z}^{r}} f(r \leq N-m)$, then $T_{\varphi}$ is hyponormal if and only if $T_{\tilde{\varphi}}$ is.

(iii) If $\left|a_{-m}\right|=\left|a_{N}\right|$, then $T_{\varphi}$ is hyponormal if and only if the following symmetric condition holds:

$$
\overline{a_{N}}\left(\begin{array}{c}
a_{-1} \\
a_{-2} \\
\vdots \\
a_{-m}
\end{array}\right)=a_{-m}\left(\begin{array}{c}
\overline{a_{N-m+1}} \\
\overline{a_{N-m+2}} \\
\vdots \\
\overline{a_{N}}
\end{array}\right) .
$$

In this case, the rank of $\left[T_{\varphi}^{*}, T_{\varphi}\right]$ is $N-m$ and $\mathcal{E}(\varphi)=\left\{a_{-m}\left(\overline{a_{N}}\right)^{-1} z^{N-m}\right\}$. In particular, $T_{\varphi}$ is normal if and only if $m=N,\left|a_{-m}\right|=\left|a_{N}\right|$, and the above symmetric condition holds.

Proof. The proof of (i) is known from [FL2], [IW] and [Zhu], the proof of (ii) is given in $\mathrm{CuL}$, and the proof of (iii) is given in [FL1].

The assertion (ii) of Proposition 1 shows that if $\varphi(z)=\sum_{n=-m}^{N} a_{n} z^{n}$, where $m \leq N, a_{-m} \neq 0$ and $a_{N} \neq 0$, then the hyponormality of $T_{\varphi}$ can be determined by that of $T_{\psi}$ with symbol $\psi$ of the form

$$
\psi(z):=\sum_{n=-m}^{m} b_{n} z^{n}, \quad \text { where } b_{n}= \begin{cases}a_{n} & (-m \leq n \leq 0) \\ a_{N-m+n} & (1 \leq n \leq m)\end{cases}
$$

In the sequel we will assume that $m \leq N$.

Our main theorem now follows:

Theorem 1. Suppose that $\varphi(z)=\sum_{n=-m}^{N} a_{n} z^{n}$, where $a_{-m}$ and $a_{N}$ are nonzero and that $c_{0}, \cdots, c_{N-1}$ are given by (3). Let $H$ denote the block Hankel matrix given 
by

$$
H:=\left(\begin{array}{ccccc}
0 & \ldots & \ldots & 0 & A_{0} \\
\vdots & & 0 & A_{0} & A_{1} \\
\vdots & . & . & . & \vdots \\
0 & A_{0} & . & & A_{m-3} \\
A_{0} & A_{1} & \ldots & A_{m-3} & A_{m-2}
\end{array}\right)
$$

where

$$
A_{j}:=\left(\begin{array}{cc}
\operatorname{Re} c_{N-m+j} & \operatorname{Im} c_{N-m+j} \\
\operatorname{Im} c_{N-m+j} & -\operatorname{Re} c_{N-m+j}
\end{array}\right) \quad \text { for } j=0, \cdots, m-2
$$

and let

$$
V:=\left(\begin{array}{c}
\operatorname{Re} c_{N-m+1} \\
\operatorname{Im} c_{N-m+1} \\
\operatorname{Re} c_{N-m+2} \\
\operatorname{Im} c_{N-m+2} \\
\vdots \\
\operatorname{Re} c_{N-1} \\
\operatorname{Im} c_{N-1}
\end{array}\right) \in \mathbb{R}^{2 m-2}
$$

If the linear system

$$
\left(I_{2 m-2}-H\right) X=V \quad \text { (the unknown is } X \in \mathbb{R}^{2 m-2} \text { ) }
$$

is solvable, let $f$ denote the analytic polynomial

$$
f(z):=c_{N-m}+\sum_{j=1}^{m-1}\left(x_{j}+i y_{j}\right) z^{j}+z^{m},
$$

where $X:=\left(x_{1}, y_{1}, x_{2}, y_{2}, \cdots, x_{m-1}, y_{m-1}\right)^{T}$ is a solution of the system (5). Then the following statements are equivalent.

1. $T_{\varphi}$ is a hyponormal operator.

2. The linear system (5) is solvable, and for every zero $\zeta$ of $f$ such that $|\zeta|>1$, the number $1 / \bar{\zeta}$ is a zero of $f$ in the open unit disk $\mathbb{D}$ of multiplicity greater than or equal to the multiplicity of $\zeta$.

In the cases where $T_{\varphi}$ is a hyponormal operator, we have that $z^{N-m} \frac{f}{z^{m} \bar{f}}$ is a finite Blaschke product in $\mathcal{E}(\varphi)$. Moreover the rank of the selfcommutator of $T_{\varphi}$ is computed from the formula

$$
\operatorname{rank}\left[T_{\varphi}^{*}, T_{\varphi}\right]=N-m+Z_{\mathbb{D}}-Z_{\mathbb{C} \backslash \overline{\mathbb{D}}},
$$

where $Z_{\mathbb{D}}$ and $Z_{\mathbb{C} \backslash \overline{\mathbb{D}}}$ are the number of zeros of $f$ in $\mathbb{D}$ and in $\mathbb{C} \backslash \overline{\mathbb{D}}$ counting multiplicity.

It is interesting and surprising that the hyponormality of $T_{\varphi}$ and the rank of the selfcommutator $\left[T_{\varphi}^{*}, T_{\varphi}\right]$ are independent of the particular solutions of the system (5). 


\section{Auxiliary Lemmas}

To prove the main theorem we need two auxiliary lemmas.

Lemma 1. Suppose that $B$ is a finite Blaschke product of degree $m \leq n$ and let

$$
k(z):=e^{i \omega} \prod_{j=1}^{r} \frac{z-\zeta_{j}}{1-\overline{\zeta_{j}} z} \quad\left(r \leq n ;\left|\zeta_{j}\right| \neq 1 ; \omega \in[0,2 \pi)\right) .
$$

Suppose that $k$ satisfies the finite interpolation

$$
\widetilde{k}(j)=\widetilde{B}(j) \quad \text { for } j=0, \cdots, n-1,
$$

where $\widetilde{k}(j)$ and $\widetilde{B}(j)$ denote the $j$-th Taylor coefficients of $k$ and $B$, respectively. (Here Taylor series expansions should be understood as to be valid for

$$
|z|<1 / \max _{1 \leq j \leq r}\left\{1,\left|\zeta_{j}\right|\right\} \text {.) }
$$

Then $k$ is also a finite Blaschke product. In particular, if $m+r<2 n$, then $B=k$, and if $m=r=n$, then $\operatorname{deg}(k)=n$.

Proof. For the first assertion, it will suffice to show that all the $\zeta_{j}$ are inside the unit circle $\mathbb{T}$. Write

$$
B(z):=e^{i \theta} \prod_{j=1}^{m} \frac{z-\alpha_{j}}{1-\overline{\alpha_{j}} z} \quad\left(\left|\alpha_{j}\right|<1 \text { for } 1 \leq j \leq m\right) .
$$

If $k$ satisfies the interpolation (8), so that $B(z)-k(z)=z^{n} \sum_{j=0}^{\infty} b_{j} z^{j}$ for some $b_{j}(j=0,1, \cdots)$, which is valid for $|z|<1 / \max _{1 \leq j \leq r}\left\{1,\left|\zeta_{j}\right|\right\}$, then multiplying $\prod_{j=1}^{m}\left(1-\overline{\alpha_{j}} z\right) \prod_{j=1}^{r}\left(1-\overline{\zeta_{j}} z\right)$ on both sides gives

$$
e^{i \theta} \prod_{j=1}^{m}\left(z-\alpha_{j}\right) \prod_{j=1}^{r}\left(1-\overline{\zeta_{j}} z\right)-e^{i \omega} \prod_{j=1}^{r}\left(z-\zeta_{j}\right) \prod_{j=1}^{m}\left(1-\overline{\alpha_{j}} z\right)=\sum_{j=n}^{m+r} d_{j} z^{j}
$$

for some $d_{j}(n \leq j \leq m+r)$. Note that the expression (9) is valid throughout $\mathbb{C}$. Observe that if $m+r<n$ then evidently, $B=k$. Thus we assume $m+r \geq n$. Write

$$
f(z):=\prod_{j=1}^{m}\left(z-\alpha_{j}\right) \prod_{j=1}^{r}\left(1-\overline{\zeta_{j}} z\right) .
$$

Suppose now that $z$ lies on the unit circle $\mathbb{T}$. Then

$$
z^{m+r} \overline{f(z)}=\prod_{j=1}^{r}\left(z-\zeta_{j}\right) \prod_{j=1}^{m}\left(1-\overline{\alpha_{j}} z\right) .
$$

Note that if $f(z):=\sum_{j=0}^{m+r} a_{j} z^{j}$, then $z^{m+r} \overline{f(z)}=\sum_{j=0}^{m+r} \overline{a_{m+r-j}} z^{j}$. Thus (9) implies that

$$
e^{i \theta} f(z)-e^{i \omega} z^{m+r} \overline{f(z)}=\sum_{j=n}^{m+r} d_{j} z^{j}
$$

so

$$
e^{i \theta} a_{j}=e^{i \omega} \overline{a_{m+r-j}} \quad(j=0, \cdots, n-1) .
$$


Therefore if $m+r<2 n$ then $e^{i \theta} a_{j}=e^{i \omega} \overline{a_{m+r-j}}$ for $j=0, \cdots, m+r$, so that $e^{i \theta} f(z)=e^{i \omega} z^{m+r} \overline{f(z)}$. This shows that again $B=k$. Now it remains to show that $\left|\zeta_{j}\right|<1$ for $j=1, \cdots, r$ when $m=r=n$. By (10) we have that

$$
e^{i \theta} f(z)-e^{i \omega} z^{2 n} \overline{f(z)}=\left(e^{i \theta} a_{n}-e^{i \omega} \overline{a_{n}}\right) z^{n} .
$$

If $e^{i \theta} a_{n}=e^{i \omega} \overline{a_{n}}$ then again $B=k$. Thus we suppose $e^{i \theta} a_{n} \neq e^{i \omega} \overline{a_{n}}$. Observe that

$$
1=\left|\frac{e^{i \theta} f(z)}{e^{i \omega} z^{2 n} \overline{f(z)}}\right|=\left|1+\frac{\left(e^{i(\theta-\omega)} a_{n}-\overline{a_{n}}\right) z^{n}}{z^{2 n} \overline{f(z)}}\right| .
$$

If we let $g(z)$ denote the function

$$
g(z):=\frac{\left(e^{i(\theta-\omega)} a_{n}-\overline{a_{n}}\right) z^{n}}{z^{2 n} \overline{f(z)}}=\frac{\left(e^{i(\theta-\omega)} a_{n}-\overline{a_{n}}\right) z^{n}}{\prod_{j=1}^{n}\left(z-\zeta_{j}\right) \prod_{j=1}^{n}\left(1-\overline{\alpha_{j}} z\right)},
$$

then by $(11), g(\mathbb{T})$ should lie in the left half-plane $\operatorname{Re} z<0$. Thus the image curve $g(\mathbb{T})$ of the unit circle $\mathbb{T}$ under $g(z)$ does not surround the origin, so that the winding number, wind $(g(\mathbb{T}), 0)$, of $g(\mathbb{T})$ with respect to 0 must be zero. But since

$$
\begin{aligned}
0 & =\text { wind }(g(\mathbb{T}), 0)=\sharp(\text { zeros of } g \text { inside } \mathbb{T})-\sharp(\text { poles of } g \text { inside } \mathbb{T}) \\
& \left.=n-\sharp\left(\text { the } \zeta_{j} \text { 's inside } \mathbb{T}\right) \quad \text { (because all the } \alpha_{j} \text { are inside } \mathbb{T}\right),
\end{aligned}
$$

it follows that $\left|\zeta_{j}\right|<1$ for all $j(1 \leq j \leq n)$. This proves the first assertion. The second assertion was already proved in the above argument. This completes the proof.

We review here Carathéodory's theorem (cf. Ga. Theorem I.2.1]) which states that for every function $k$ in the closed unit ball of $H^{\infty}(\mathbb{T})$ there exists a sequence $\left\{B_{n}\right\}$ of finite Blaschke products that converges to $k(z)$ pointwise on $\mathbb{D}$. Its proof relies upon a construction of a sequence $\left\{B_{n}\right\}$ of finite Blaschke products satisfying that if $k(z)=\sum_{j=0}^{\infty} c_{j} z^{j}$ is in the closed unit ball of $H^{\infty}(\mathbb{T})$, then

$$
\widehat{B_{n}}(j)=c_{j} \quad \text { for } j=0, \cdots, n, \quad\left(\widehat{B_{n}}(j) \text { denotes the } j \text {-th Fourier coefficient of } B_{n}\right) \text {. }
$$

The construction runs as follows. Write $\Phi_{n}$ for the $n$-th Schur's function corresponding to the function $k$. Since $\left|\Phi_{0}\right|=\left|c_{0}\right| \leq 1$, we can take $B_{0}:=\frac{z+\Phi_{0}}{1+\overline{\Phi_{0}} z}$. If $\left|\Phi_{0}\right|=1$ then $B_{0}=c_{0}$ is the Blaschke product such that $B_{0}=k$. Write $B_{0}^{(0)}:=B_{0}$. If $\left|\Phi_{j}\right|<1$ for $j=0, \cdots, n$, let

$$
B_{n}^{(0)}:=\frac{z+\Phi_{n}}{1+\overline{\Phi_{n}} z}
$$

and define by induction

$$
B_{n}^{(j)}:=\frac{z B_{n}^{(j-1)}+\Phi_{n-j}}{1+\bar{\Phi}_{n-j} z B_{n}^{(j-1)}} \quad(j=1, \cdots, n) .
$$

Set $B_{n}:=B_{n}^{(n)}$. Then $B_{n}$ satisfies the interpolation $\widehat{B_{n}}(j)=c_{j}$ for $j=0, \cdots, n$. If $\left|\Phi_{n}\right|=1$ then $B_{n}$ is the finite Blaschke product such that $B_{n}=k$. This will be referred to as the Carathéodory construction.

Lemma 2. Suppose that $\varphi(z)=\sum_{n=-m}^{m} a_{n} z^{n}$, where $a_{-m}$ and $a_{m}$ are nonzero, is such that $T_{\varphi}$ is hyponormal. Then the following statements are equivalent.

1. $\operatorname{rank}\left[T_{\varphi}^{*}, T_{\varphi}\right]=r$.

2. $\left|\Phi_{r}\right|=1$ if $r \leq m-1 ; \quad\left|\Phi_{m-1}\right|<1$ if $r=m$. 
3. There exists an analytic polynomial $f$ of degree $m$ with the leading coefficient 1 such that $\frac{f}{z^{m} \bar{f}}$ is a finite Blaschke product in $\mathcal{E}(\varphi)$ of degree $r$.

Remark. If $f(z)=\prod_{j=1}^{m}\left(z-\zeta_{j}\right)$, then $z^{m} \overline{f(z)}=\prod_{j=1}^{m}\left(1-\overline{\zeta_{j}} z\right)$ on $\mathbb{T}$. Thus if $\frac{f}{z^{m} \bar{f}}$ is in $\mathcal{E}(\varphi)$, then $\frac{f}{z^{m} \bar{f}}$ is a finite Blaschke product of degree at most $m$. In fact, if $\operatorname{rank}\left[T_{\varphi}^{*}, T_{\varphi}\right]=r$, then by the Nakazi-Takahashi theorem there exists a finite Blaschke product $k \in \mathcal{E}(\varphi)$ of the form

$$
k(z)=e^{i \theta} \prod_{j=1}^{r} \frac{z-\beta_{j}}{1-\overline{\beta_{j}} z} \quad\left(\left|\beta_{j}\right|<1 \text { for } j=1, \cdots, r\right) .
$$

Therefore the crucial point of Lemma 2 is that if $r=m$, then we can choose $\theta=0$ in (12) (see the proof below).

Proof of Lemma 2. (2) $\Rightarrow$ (3): Suppose that $k(z)=\sum_{j=0}^{\infty} c_{j} z^{j}$ is in $\mathcal{E}(\varphi)$, so $c_{0}, \cdots, c_{m-1}$ are given by (3) with $N=m$. Thus we must find a Blaschke product $B$ of degree $r$ such that $B(z):=\frac{f(z)}{z^{m} \overline{f(z)}}$ whose first $m$ coefficients match those of $k$, where $f$ is an analytic polynomial of degree $m$ with the leading coefficient 1 . To do this we will use the Carathéodory construction. Let $\left\{B_{n}\right\}$ be the sequence of finite Blaschke products in the Carathéodory construction. We first claim that if $\left|\Phi_{n}\right|<1$ (and hence $\left|\Phi_{j}\right|<1$ for $j=0, \cdots, n-1$ ), then

$$
B_{n}=\frac{f}{z^{n+1} \bar{f}} \quad \text { with } \operatorname{deg}\left(B_{n}\right)=n+1,
$$

where $f$ is an analytic polynomial of degree $n+1$ with the leading coefficient 1 . For (13) we use an induction argument on $j$. Evidently, $B_{n}^{(0)}=\frac{z+\Phi_{n}}{1+\bar{\Phi}_{n} z}$ is a Blaschke product of degree 1. If $f_{0}:=z+\Phi_{n}$ then $f_{0}$ is a polynomial of degree 1 with the leading coefficient 1 and $B_{n}^{(0)}=\frac{f_{0}}{z \overline{f_{0}}}$. Suppose that $B_{n}^{(j-1)}=\frac{f_{j-1}}{z^{j} \overline{f_{j-1}}}$ is a Blaschke product of degree $j$ and $f_{j-1}$ is an analytic polynomial of degree $j$ with the leading coefficient 1 . Note that the number of zeros of $f_{j-1}$ in $\mathbb{D}$ is $j$. Observe that

$$
B_{n}^{(j)}(z)=\frac{z B_{n}^{(j-1)}(z)+\Phi_{n-j}}{1+\bar{\Phi}_{n-j} z B_{n}^{(j-1)}(z)}=\frac{z f_{j-1}(z)+\Phi_{n-j} z^{j} \overline{f_{j-1}(z)}}{z^{j} \overline{f_{j-1}(z)}+\overline{\Phi_{n-j}} z f_{j-1}(z)} .
$$

If we define $f_{j}(z):=z f_{j-1}(z)+\Phi_{n-j} z^{j} \overline{f_{j-1}(z)}$, then $B_{n}^{(j)}=\frac{f_{j}}{z^{j+1} \overline{f_{j}}}$ and by the inductive hypothesis, $f_{j}$ is an analytic polynomial of degree $j+1$ with the leading coefficient 1. Concerning the degree of $B_{n}^{(j)}$, note that $\left|\Phi_{n-j}\right|<1$. We need to show that the number of zeros of $f_{j}$ in $\mathbb{D}$ is $j+1$. Observe that

$$
\left|\Phi_{n-j} z^{j} \overline{f_{j-1}(z)}\right|=\left|\Phi_{n-j}\right|\left|f_{j-1}(z)\right|<\left|f_{j-1}(z)\right|=\left|z f_{j-1}(z)\right| \text { on } \mathbb{T} \text {. }
$$

Therefore by Rouché's theorem and the inductive hypothesis,

$$
\sharp\left(\text { zeros of } f_{j} \text { in } \mathbb{D}\right)=\sharp\left(\text { zeros of } z f_{j-1} \text { in } \mathbb{D}\right)=j+1 \text {, }
$$

which implies that $\operatorname{deg}\left(B_{n}^{(j)}\right)=j+1$. This proves (13). Therefore if $\left|\Phi_{m-1}\right|<1$ then by (13), $B_{m-1}$ is the required Blaschke product in $\mathcal{E}(\varphi)$. If instead $\left|\Phi_{r}\right|=1$ for $r \leq m-1$ (and hence $\left|\Phi_{j}\right|<1$ for $j=0, \cdots, r-1$ ), then we claim that $B_{r}$ is a Blaschke product in $\mathcal{E}(\varphi)$ of degree $r$. Indeed,

$$
B_{r}^{(0)}=\frac{z+\Phi_{r}}{1+\overline{\Phi_{r}} z}=\Phi_{r}=: e^{i \omega} \quad \text { for some } \omega \in[0,2 \pi) .
$$


So

$$
B_{r}^{(1)}=\frac{z e^{i \omega}+\Phi_{r-1}}{1+\overline{\Phi_{r-1}} z e^{i \omega}}=e^{i \omega} \frac{z+e^{-i \omega} \Phi_{r-1}}{1+\overline{e^{-i \omega} \Phi_{r-1}} z}
$$

is a Blaschke product of degree 1. By the same argument as the above we can see that $B_{r}=B_{r}^{(r)}$ is a Blaschke product in $\mathcal{E}(\varphi)$ of degree $r$. Thus we can write

$$
B_{r}(z)=e^{i \omega} \prod_{j=1}^{r} \frac{z-\zeta_{j}}{1-\overline{\zeta_{j}} z} \quad\left(\left|\zeta_{j}\right|<1 \text { for } j=1, \cdots, r\right) .
$$

If we take

$$
f(z):=\prod_{j=1}^{r}\left(z-\zeta_{j}\right) \prod_{j=1}^{m-r}\left(z+e^{i\left(\frac{\omega}{m-r}\right)}\right),
$$

then $B_{r}(z)=\frac{f(z)}{z^{m} \overline{f(z)}}$ is the required Blaschke product of degree $r$.

$(3) \Rightarrow(1)$ : Suppose that $\frac{f}{z^{m} \bar{f}}$ is a finite Blaschke product in $\mathcal{E}(\varphi)$ of degree $r$. Assume to the contrary that $\operatorname{rank}\left[T_{\varphi}^{*}, T_{\varphi}\right]=\ell \neq r$. Then by the Nakazi-Takahashi theorem we can find a finite Blaschke product $B$ of degree $\ell$ in $\mathcal{E}(\varphi)$. Thus by the second assertion of Lemma 1 , we have that $B=\frac{f}{z^{m} \bar{f}}$, a contradiction.

(1) $\Rightarrow(2)$ : If $\operatorname{rank}\left[T_{\varphi}^{*}, T_{\varphi}\right]=r \leq m-1$, then by the Nakazi-Takahashi theorem, there exists a finite Blaschke product $B_{1}$ in $\mathcal{E}(\varphi)$ of degree $r$. Assume to the contrary that $\left|\Phi_{r}\right|<1$. Then in view of $(13)$, we can find a finite Blaschke product $B_{2}$ in $\mathcal{E}(\varphi)$ such that $r+1 \leq \operatorname{deg}\left(B_{2}\right) \leq m$. But by the second assertion of Lemma 1, we have that $B_{1}=B_{2}$, a contradiction. Therefore we have that $\left|\Phi_{r}\right|=1$. If instead $\operatorname{rank}\left[T_{\varphi}^{*}, T_{\varphi}\right]=m$ then by the same argument we must have that $\left|\Phi_{m-1}\right|<1$.

\section{Proof of the Main Theorem}

We are ready to prove Theorem 1.

Proof of Theorem 1. In view of the assertion (ii) of Proposition 1 we may assume without loss of generality that $N=m$ for the hyponormality of $T_{\varphi}$. Let $f$ be an analytic polynomial of the form $f(z):=\sum_{j=0}^{m} b_{j} z^{j}$ with $b_{m}=1$. Then for all $z$ on the unit circle $\mathbb{T}, z^{m} \overline{f(z)}=\sum_{j=0}^{m} \overline{b_{m-j}} z^{j}$. Thus $\frac{f}{z^{m} \bar{f}} \in \mathcal{E}(\varphi)$ if and only if the following two conditions are satisfied:

(a) $\frac{f}{z^{m} \bar{f}}$ satisfies the interpolation

$$
\widetilde{\left(\frac{f}{z^{m} \bar{f}}\right)}(j)=c_{j} \quad(j=0, \cdots, m-1),
$$

where the $c_{j}$ are given by (3) with $N=m$;

(b) $\frac{f}{z^{m} \bar{f}} \in H^{\infty}(\mathbb{T})$.

On the other hand, the interpolation (14) is solvable if and only if

$$
\sum_{j=0}^{m} b_{j} z^{j}=\left(\sum_{j=0}^{m} \overline{b_{m-j}} z^{j}\right)\left(\sum_{j=0}^{m-1} c_{j} z^{j}+\sum_{j=m}^{\infty} d_{j} z^{j}\right) \quad \text { for some } d_{j}(j \geq m)
$$


or in matrix form,

$$
\left(\begin{array}{c}
b_{0} \\
b_{1} \\
\vdots \\
b_{m-1} \\
b_{m}
\end{array}\right)=\left(\begin{array}{ccccc} 
& & & & c_{0} \\
0 & & c_{0} & c_{1} \\
& & . & . & \vdots \\
& c_{0} & c_{1} & \ldots & c_{m-1} \\
c_{0} & c_{1} & \ldots & c_{m-1} & d_{m}
\end{array}\right)\left(\begin{array}{c}
\overline{b_{0}} \\
\overline{b_{1}} \\
\vdots \\
\overline{b_{m-1}} \\
\overline{b_{m}}
\end{array}\right) \quad \text { for some } d_{m}
$$

If we write

$$
\begin{gathered}
b_{j}:=x_{j}+i y_{j}(0 \leq j \leq m), \quad c_{j}:=\alpha_{j}+i \beta_{j}(0 \leq j \leq m-1), \quad \text { and } \\
d_{m}:=\alpha_{m}+i \beta_{m}
\end{gathered}
$$

for the rectangular representation of each entry in (16), then the system (16) is solvable if and only if the following system is solvable for some $\alpha_{m}, \beta_{m} \in \mathbb{R}$ :

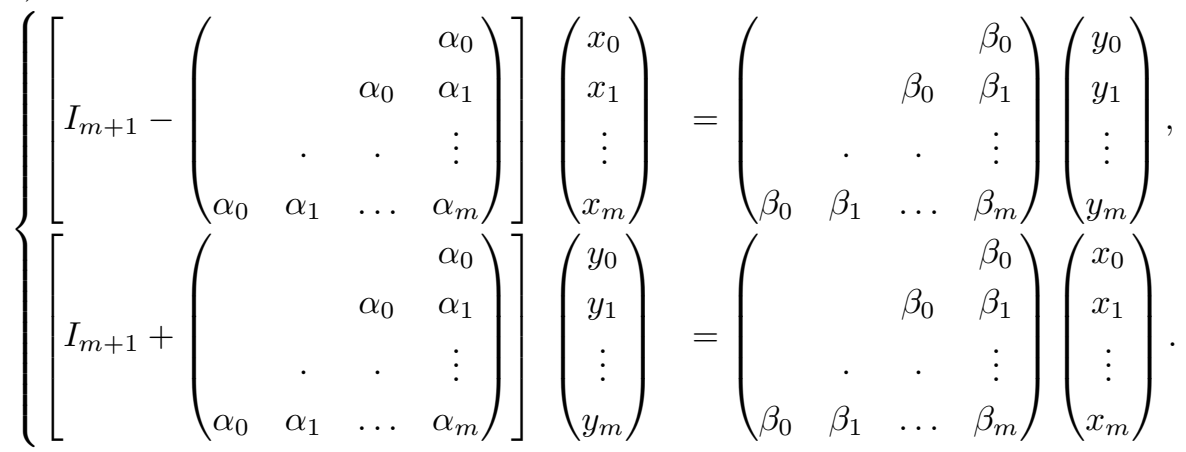

Note that $x_{m}=1$ and $y_{m}=0$. Thus a simplification of (17) shows that the system (17) is solvable if and only if $b_{0}=c_{0}, b_{m}=1$, and the following system is solvable:

$$
\left(I_{2 m-2}-H\right) X=V,
$$

where

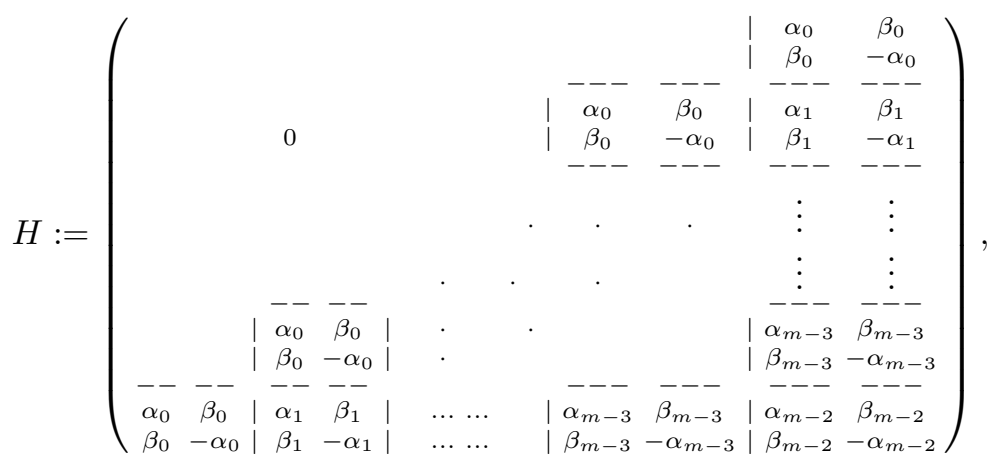




$$
X:=\left(\begin{array}{c}
x_{1} \\
y_{1} \\
x_{2} \\
y_{2} \\
\vdots \\
\vdots \\
x_{m-1} \\
y_{m-1}
\end{array}\right) \quad \text { and } \quad V:=\left(\begin{array}{c}
\alpha_{1} \\
\beta_{1} \\
\alpha_{2} \\
\beta_{2} \\
\vdots \\
\vdots \\
\alpha_{m-1} \\
\beta_{m-1}
\end{array}\right)
$$

Therefore if the system (18) is solvable and if $\frac{f}{z^{m} \bar{f}} \in H^{\infty}(\mathbb{T})$, then $\frac{f}{z^{m} \bar{f}} \in \mathcal{E}(\varphi)$ and hence $T_{\varphi}$ is hyponormal. But note that $\zeta$ is a zero of $f$ if and only if $1 / \bar{\zeta}$ is a zero of $z^{m} \bar{f}$. Therefore $\frac{f(z)}{z^{m} \overline{f(z)}}$ is analytic in $\mathbb{D}$ if and only if the zeros of $f$ have the property that for every zero $\zeta$ of $f$ with $|\zeta|>1$, the complex number $1 / \bar{\zeta}$ is a zero of $f$ in $\mathbb{D}$ of multiplicity greater than or equal to the multiplicity of $\zeta$. This proves the sufficient condition for the hyponormality of $T_{\varphi}$. Towards the necessity condition, suppose that $T_{\varphi}$ is hyponormal. Then by Lemma 2 there exists an analytic polynomial $f$ of the form $f(z)=\sum_{j=0}^{m} b_{j} z^{j}$ with $b_{m}=1$ such that $\frac{f}{z^{m} \bar{f}} \in \mathcal{E}(\varphi)$. Thus by the preceding argument the system (18) is solvable. It remains to show that all solutions of the system (18) satisfy the second condition of statement (2) of this theorem. This follows at once from Lemma 1: if $g$ is an analytic polynomial induced by another solution of the system (18), then $\frac{g}{z^{m} \bar{g}}$ must satisfy the interpolation

$$
\widetilde{\left(\frac{f}{z^{m} \bar{f}}\right)}(j)=\widetilde{\left(\frac{g}{z^{m} \bar{g}}\right)}(j) \quad \text { for } j=0, \cdots, m-1 .
$$

Thus by Lemma $1, \frac{g}{z^{m} \bar{g}}$ is also a finite Blaschke product, which implies that $g$ also satisfies the second condition of statement (2) of this theorem. This proves the criterion which was sought.

For the second assertion, we argue that if $\varphi(z)=\sum_{n=-m}^{N} a_{n} z^{n}(m \leq N)$ and if $\psi(z)=\sum_{n=-m}^{m} b_{n} z^{n}$ is the corresponding induced trigonometric polynomial as in (4), then

$$
\operatorname{rank}\left[T_{\varphi}^{*}, T_{\varphi}\right]=N-m+\operatorname{rank}\left[T_{\psi}^{*}, T_{\psi}\right] .
$$

Indeed if $B_{\varphi}$ and $B_{\psi}$ are the corresponding Blaschke products in $\mathcal{E}(\varphi)$ and $\mathcal{E}(\psi)$ such that $\operatorname{rank}\left[T_{\varphi}^{*}, T_{\varphi}\right]=\operatorname{deg}\left(B_{\varphi}\right)$ and $\operatorname{rank}\left[T_{\psi}^{*}, T_{\psi}\right]=\operatorname{deg}\left(B_{\psi}\right)$ then we can find $B_{\varphi}$ and $B_{\psi}$ satisfying $B_{\varphi}=z^{N-m} B_{\psi}$, which implies (19). Thus if $T_{\psi}$ is hyponormal then by the preceding argument, $B:=\frac{f}{z^{m} \bar{f}}$ is a finite Blaschke product in $\mathcal{E}(\psi)$ of degree at most $m$, which implies that $z^{N-m} \frac{f}{z^{m} \bar{f}} \in \mathcal{E}(\varphi)$. In view of Lemma 1 , the degree of $B$ is independent of the particular choices of $f$. If $\operatorname{deg}(B)<m$ then by the Nakazi-Takahashi theorem and the second assertion of Lemma 1, we have that $\operatorname{rank}\left[T_{\psi}^{*}, T_{\psi}\right]=\operatorname{deg}(B)$. If instead $\operatorname{deg}(B)=m$ then evidently, $\operatorname{rank}\left[T_{\psi}^{*}, T_{\psi}\right]=m$. Therefore if $Z_{\mathbb{D}}$ and $Z_{\mathbb{C} \backslash \overline{\mathbb{D}}}$ denote the number of zeros of $f$ in $\mathbb{D}$ and in $\mathbb{C} \backslash \overline{\mathbb{D}}$ counting multiplicity, then since $\operatorname{deg}(B)=Z_{\mathbb{D}}-Z_{\mathbb{C} \backslash \mathbb{\mathbb { D }}}$, it follows that $\operatorname{rank}\left[T_{\varphi}^{*}, T_{\varphi}\right]=$ $N-m+Z_{\mathbb{D}}-Z_{\mathbb{C} \backslash \overline{\mathbb{D}}}$. This completes the proof. 


\section{Remarks AND Examples}

Remark 1. In Lemma 2, the statement (3) cannot be strengthened by adding the requirement that there exists an analytic polynomial $f$ of degree $r$ with the leading coefficient 1 such that $\frac{f}{z^{r} \bar{f}}$ is a finite Blaschke product in $\mathcal{E}(\varphi)$ of degree $r$. To see this consider the trigonometric polynomial

$$
\varphi(z)=z^{-2}+2 z^{-1}+i z+2 i z^{2} .
$$

Then a straightforward calculation shows that $\operatorname{rank}\left[T_{\varphi}^{*}, T_{\varphi}\right]=1$ and $\mathcal{E}(\varphi)$ has precisely one element $i \frac{z+\frac{1}{2}}{1+\frac{1}{2} z}$. This illustrates the above assertion.

Remark 2. If $\varphi(z)=\sum_{n=-m}^{m} a_{n} z^{n}$ with $\left|a_{m}\right|=\left|a_{-m}\right|$, then $\left|c_{0}\right|=1$ because $c_{0}=$ $\frac{a_{-m}}{\overline{a_{m}}}$. So if $T_{\varphi}$ is hyponormal then $c_{1}=\cdots=c_{m-1}=0$. Thus $f(z)=c_{0}+$ $z^{m}$ satisfies the two conditions in statement (2) of Theorem 1. Note that $f$ has zeros only on the unit circle $\mathbb{T}$, so that $\operatorname{rank}\left[T_{\varphi}^{*}, T_{\varphi}\right]=0$ and hence $T_{\varphi}$ is normal. Therefore we can conclude that if $\left|a_{m}\right|=\left|a_{-m}\right|$, then $T_{\varphi}$ is hyponormal if and only if $T_{\varphi}$ is normal; this recaptures the key point in the assertion (iii) of Proposition 1.

Example 1. Consider the trigonometric polynomial

$$
\varphi(z)=-2 z^{-4}+9 z^{-3}-12 z^{-2}+4 z^{-1}-2 z^{2}+9 z^{3}-12 z^{4}+4 z^{5} .
$$

We use Theorem 1 to determine the hyponormality of $T_{\varphi}$. Observe that $c_{0}=0$ and

$$
\left(\begin{array}{c}
\overline{c_{1}} \\
\frac{c_{2}}{\overline{c_{3}}} \\
\frac{c_{4}}{4}
\end{array}\right)=\left(\begin{array}{cccc}
-2 & 9 & -12 & 4 \\
9 & -12 & 4 & 0 \\
-12 & 4 & 0 & 0 \\
4 & 0 & 0 & 0
\end{array}\right)^{-1}\left(\begin{array}{c}
4 \\
-12 \\
9 \\
-2
\end{array}\right)=\left(\begin{array}{c}
-\frac{1}{2} \\
\frac{3}{4} \\
\frac{3}{8} \\
\frac{3}{16}
\end{array}\right)
$$

and in turn,

$$
I_{6}-H=\left(\begin{array}{cccccc}
1 & 0 & 0 & 0 & \frac{1}{2} & 0 \\
0 & 1 & 0 & 0 & 0 & -\frac{1}{2} \\
0 & 0 & \frac{3}{2} & 0 & -\frac{3}{4} & 0 \\
0 & 0 & 0 & \frac{1}{2} & 0 & \frac{3}{4} \\
\frac{1}{2} & 0 & -\frac{3}{4} & 0 & \frac{5}{8} & 0 \\
0 & -\frac{1}{2} & 0 & \frac{3}{4} & 0 & \frac{11}{8}
\end{array}\right) \quad \text { and } \quad V=\left(\begin{array}{c}
\frac{3}{4} \\
0 \\
\frac{3}{8} \\
0 \\
\frac{3}{16} \\
0
\end{array}\right)
$$

Since $\operatorname{rank}\left[I_{6}-H\right]=4=\operatorname{rank}\left[I_{6}-H: V\right]$, the system (5) is solvable and has two free variables. Set $x_{2}=y_{2}=0$. Then the solution of the system is given by $x_{1}=1$, $x_{3}=-\frac{1}{2}, y_{1}=y_{3}=0$. Thus the testing polynomial $f$ is obtained by

$$
f(z)=-\frac{1}{2}+z-\frac{1}{2} z^{3}+z^{4}
$$

which has zeros at $z=\frac{1}{2},-1,(-1)^{\frac{1}{3}},-(-1)^{\frac{2}{3}}$. Therefore by Theorem $1, T_{\varphi}$ is hyponormal, and $\operatorname{rank}\left[T_{\varphi}^{*}, T_{\varphi}\right]=N-m+Z_{\mathbb{D}}-Z_{\mathbb{C} \backslash \overline{\mathbb{D}}}=5-4+1=2$. Moreover,

$$
z \frac{z-\frac{1}{2}}{1-\frac{1}{2} z} \in \mathcal{E}(\varphi) \text {. }
$$

To illustrate that other solutions of the system (5) lead to the same result, let us take $x_{3}=y_{3}=0$. Then the solution of the system (5) is given by $x_{1}=\frac{3}{4}$, $x_{2}=\frac{1}{4}, y_{1}=y_{2}=0$. So $f(z)=-\frac{1}{2}+\frac{3}{4} z+\frac{1}{4} z^{2}+z^{4}$, which has zeros at $z=$ $\frac{1}{2},-1, \frac{1}{4}(1-\sqrt{15} i), \frac{1}{4}(1+\sqrt{15} i)$, which leads to the same result as the above. 
Example 2. Consider the trigonometric polynomial

$$
\varphi(z)=z^{-4}+z^{-3}+2 z^{-2}+z^{2}+2 z^{3}+2 z^{4} .
$$

Observe that

$$
\left(\begin{array}{l}
\overline{c_{0}} \\
\frac{\bar{c}}{c_{2}} \\
\frac{c_{3}}{c_{3}}
\end{array}\right)=\left(\begin{array}{llll}
0 & 1 & 2 & 2 \\
1 & 2 & 2 & 0 \\
2 & 2 & 0 & 0 \\
2 & 0 & 0 & 0
\end{array}\right)^{-1}\left(\begin{array}{l}
0 \\
2 \\
1 \\
1
\end{array}\right)=\left(\begin{array}{c}
\frac{1}{2} \\
0 \\
\frac{3}{4} \\
-\frac{3}{4}
\end{array}\right)
$$

and in turn,

$$
I_{6}-H=\left(\begin{array}{cccccc}
1 & 0 & 0 & 0 & -\frac{1}{2} & 0 \\
0 & 1 & 0 & 0 & 0 & \frac{1}{2} \\
0 & 0 & \frac{1}{2} & 0 & 0 & 0 \\
0 & 0 & 0 & \frac{3}{2} & 0 & 0 \\
-\frac{1}{2} & 0 & 0 & 0 & \frac{1}{4} & 0 \\
0 & \frac{1}{2} & 0 & 0 & 0 & \frac{7}{4}
\end{array}\right) \quad \text { and } \quad V=\left(\begin{array}{c}
0 \\
0 \\
\frac{3}{4} \\
0 \\
-\frac{3}{4} \\
0
\end{array}\right)
$$

Then a straightforward calculation shows that

$$
\operatorname{rank}\left[I_{6}-H\right]=5 \neq 6=\operatorname{rank}\left[I_{6}-H: V\right] .
$$

Thus the system (5) has no solution, and hence by Theorem $1, T_{\varphi}$ is not hyponormal.

\section{REFERENCES}

[BH] A. Brown and P.R. Halmos, Algebraic properties of Toeplitz operators, J. Reine Angew. Math. 213 (1963-1964), 89-102. MR 28:3350

[Co1] C.C. Cowen, Hyponormal and subnormal Toeplitz operators, Surveys of Some Recent Results in Operator Theory, I (J.B. Conway and B.B. Morrel, eds.), Pitman Research Notes in Mathematics, Vol 171, Longman, 1988, pp. 155-167. MR 90j:47022

[Co2] C.C. Cowen, Hyponormality of Toeplitz operators, Proc. Amer. Math. Soc. 103 (1988), 809-812. MR 89f: 47038

[CoL] C.C. Cowen and J.J. Long, Some subnormal Toeplitz operators, J. Reine Angew. Math. 351 (1984), 216-220. MR 86h:47034

[CuL] R.E. Curto and W.Y. Lee, Joint hyponormality of Toeplitz pairs, Memoirs Amer. Math. Soc. no. 712, Amer. Math. Soc., Providence, 2001. CMP 2001:07

[Fa] P. Fan, Remarks on hyponormal trigonometric Toeplitz operators, Rocky Mountain J. Math. 13 (1983), 489-493. MR 84j:47044

[FL1] D.R. Farenick and W.Y. Lee, Hyponormality and spectra of Toeplitz operators, Trans. Amer. Math. Soc. 348 (1996), 4153-4174. MR 97k:47027

[FL2] D.R. Farenick and W.Y.Lee, On hyponormal Toeplitz operators with polynomial and circulant-type symbols, Integral Equations Operator Theory 29 (1997), 202-210. MR 98j:47059

[Ga] J.B. Garnett, Bounded Analytic Functions, Academic Press, New York, 1981. MR 83g:30037

[Gu] C. Gu, A generalization of Cowen's characterization of hyponormal Toeplitz operators, J. Funct. Anal. 124 (1994), 135-148. MR 95j:47034

[Ha1] P.R. Halmos, Ten problems in Hilbert space, Bull. Amer. Math. Soc. 76 (1970), 887-933. MR 42:5066

[Ha2] P.R. Halmos, Ten years in Hilbert space, Integral Equations Operator Theory 2 (1979), 529-564. MR 81c: 47003

[HKL] I.S. Hwang, I.H. Kim and W.Y. Lee, Hyponormality of Toeplitz operators with polynomial symbols, Math. Ann. 313 (1999), 247-261. MR 2000a:47056

[IW] T. Ito and T.K. Wong, Subnormality and quasinormality of Toeplitz operators, Proc. Amer. Math. Soc. 34 (1972), 157-164. MR 46:2472

[NT] T. Nakazi and K. Takahashi, Hyponormal Toeplitz operators and extremal problems of Hardy spaces, Trans. Amer. Math. Soc. 338 (1993), 753-769. MR 93j:47040 
[Sch] I. Schur, Über Potenzreihen die im Innern des Einheitskreises beschränkt sind, J. Reine Angew. Math. 147 (1917), 205-232.

[Zhu] K. Zhu, Hyponormal Toeplitz operators with polynomial symbols, Integral Equations Operator Theory 21 (1995), 376-381. MR 95m:47044

Department of Mathematics, Sungkyunkwan University, Suwon 440-746, Korea

E-mail address: ishwang@math.skku.ac.kr

Department of Mathematics, Sungkyunkwan University, Suwon 440-746, Korea

E-mail address: wylee@yurim.skku.ac.kr 